\title{
GRAINE project and first results on 2018 balloon-borne experiment
}

\author{
Satoru Takahashi* \\ Kobe University \\ E-mail: satorueradix.h.kobe-u.ac.jp \\ for the GRAINE collaboration \\ Aichi University of education, ISAS/JAXA, Kobe University, Nagoya University, Okayama \\ University of science
}

\begin{abstract}
We are developing GRAINE project, $10 \mathrm{MeV}-100 \mathrm{GeV} \operatorname{cosmic} \gamma$-ray observations with a precise (0.08 degree @ $1-2 \mathrm{GeV})$ and polarization sensitive large-aperture-area $\left(\sim 10 \mathrm{~m}^{2}\right)$ emulsion telescope by repeating long duration balloon flights. In April 2018, we performed a balloonborne experiment with various developments, improvements and preparations, and with a 0.38 $\mathrm{m}^{2}$ aperture and 17.4 hour flight duration in Australia to demonstrate overall performance of the telescope with a detection and imaging of a known $\gamma$-ray source, Vela pulsar. In 2021 or later, with a $10 \mathrm{~m}^{2}$ and $>\sim 24$ hour flight duration, we aim to start scientific observations.
\end{abstract}

36th International Cosmic Ray Conference -ICRC2019-

July 24th - August 1st, 2019

Madison, WI, U.S.A.

\footnotetext{
* Speaker.
} 


\section{Introduction}

Observations of high-energy cosmic $\gamma$-rays provide us with direct information of high-energy phenomena in the universe. Fermi-LAT and other telescopes are observing a $\gamma$-ray sky and offering new insights. On the other hand, past and present observations have some limitations. Improvements in an angular resolution and polarization sensitivity are one of keys for a breakthrough in the field of $\gamma$-ray observations.

We are developing GRAINE project, $10 \mathrm{MeV}-100 \mathrm{GeV}$ cosmic $\gamma$-ray observations with a precise (0.08 degree @ $1-2 \mathrm{GeV})$ and polarization sensitive large-aperture-area $\left(\sim 10 \mathrm{~m}^{2}\right)$ emulsion telescope by repeating long duration balloon flights.

Various test experiments and developments were performed on the ground [1] 3]. In 2011, the first balloon-borne emulsion $\gamma$-ray telescope experiment was performed with a $125 \mathrm{~cm}^{2}$ aperture and 4.3 hour flight duration in Japan. In 2015, a balloon-borne experiment was performed with a $3780 \mathrm{~cm}^{2}$ aperture and 14.4 hour flight duration in Australia. We demonstrated a feasibility and performance of the balloon-borne emulsion $\gamma$-ray telescope experiments [4, 5, , 7, 8].

\section{2018 balloon-borne experiment}

In April 2018, we performed a balloon-borne experiment with various developments, improvements and preparations, and with a $0.38 \mathrm{~m}^{2}$ aperture and 17.4 hour flight duration in Australia to demonstrate overall performance of the telescope with a detection and imaging of a known $\gamma$-ray source, Vela pulsar [9]. The final assembly was done at the Alice Springs balloon-launching station in March of 2018. We checked the onboard equipment (multistage shifter, star cameras, temperature meters, pressure meters, GPS systems, and battery) and assembled them on the pressure vessel gondola. After mounting the emulsion films and rehearsing for the launch, we were ready for flight on end of March, 2018 (Fig. 1 2). After waiting for appropriate wind conditions, the balloon was launched on April 26, 2018 (Fig. 3, 4). The balloon was launched at 06:33 Australian Central Standard Time (ACST). Launched balloon was going up and reached at a $38 \mathrm{~km}$ altitude after 2 hours from the launch. Then the balloon started a level flight on west wind. Observations ended at 22:15 and the gondola was released at 23:17. The gondola landed by a parachute about $900 \mathrm{~km}$ east of Alice Springs and $250 \mathrm{~km}$ southwest of Longreach at 23:54. Fig. 5, 6 and 7 show a flight path, altitude, and residual atmospheric pressure. The total flight duration in this experiment was $17.4 \mathrm{~h}$, with nearly $15 \mathrm{~h}$ of level flight at $35-38 \mathrm{~km}$ altitude and $3-5 \mathrm{hPa}$ residual atmospheric pressure. The Vela pulsar was fully covered in 45 degree zenith above 6 hours. Fig. 8 shows an operation of multi-stage shifter. The multistage shifter was operated after the launch at a speed of $1 \mu \mathrm{m} / \mathrm{s}$ in the third stage. Starting at 14:48, the operation mode was changed to $10 \mu \mathrm{m} / \mathrm{s}$ speed in the third stage to cover the Vela pulsar in 45 degree zenith with a timing resolution of sub-second which has enough resolution to image Vela pulsar with 1 degree above $100 \mathrm{MeV}$ aimed in this experiment. The operation parameters were based on an operation in accelerator neutrino experiment, J-PARC T60 [10]. The operation ended at 22:15. Fig. 8 also shows the repeatability with which the third and second stages of the multistage shifter return to their reference positions. We stably operated the multistage shifter during the flight. Star cameras also stably operated during the flight (Fig. 9 ). Fig. 10 shows the status of the pressure vessel during the flight. The pressure vessel was worked 
during the flight [11]. We performed stable operation of the emulsion $\gamma$-ray telescope during the flight. The gondola was successfully recovered from landing point to Longreach in the next day. The emulsion films were sent from Longreach to Sydney on April 28, 2018 by ground below $\sim 10$ ${ }^{\circ} \mathrm{C}$ using a truck with a refrigerator. Post-flight emulsion film processing was done at the University of Sydney. All emulsion films were developed by May 13, 2018 with negligible loss due to development errors.

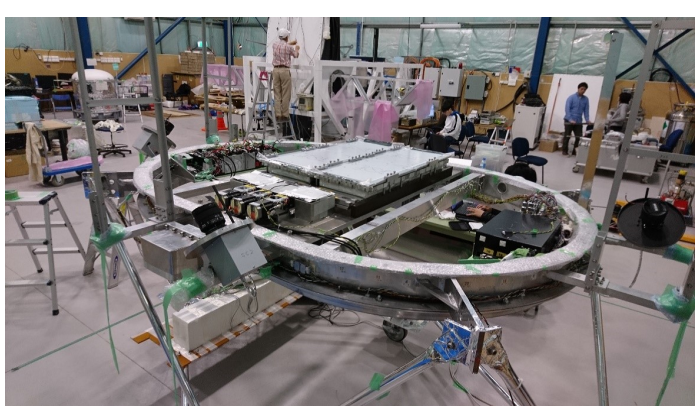

Figure 1: Gondola at final preparations.

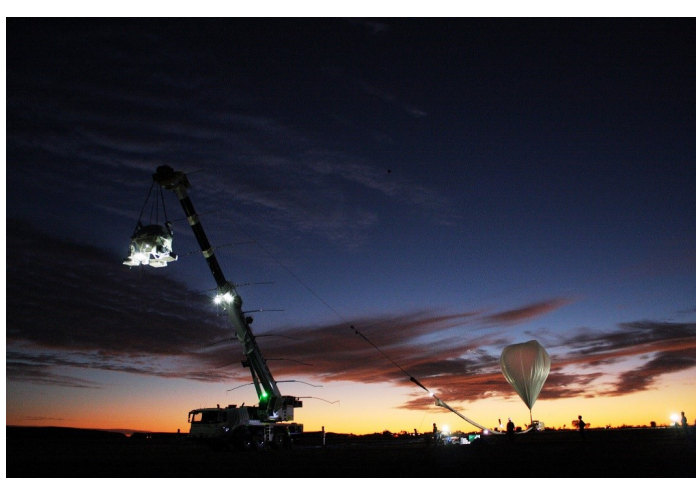

Figure 3: Before balloon launching (photo by JAXA).

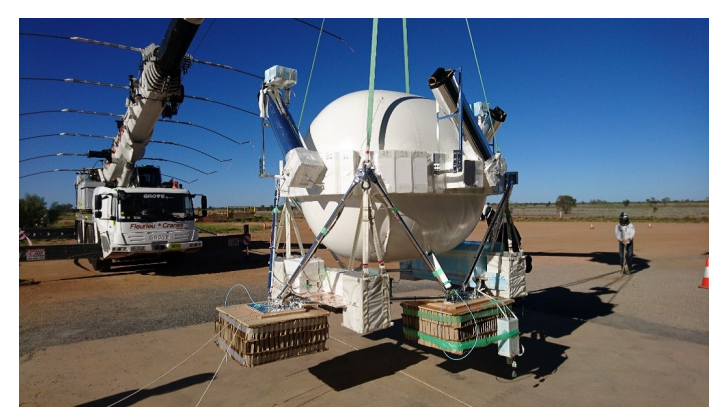

Figure 2: Gondola to be ready for the flight.

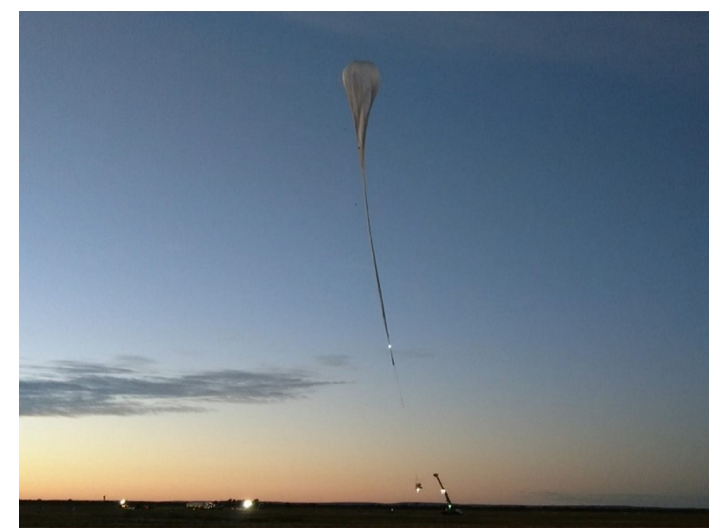

Figure 4: Balloon launching.

After getting back to Japan with the emulsion films and acquired data, we are going on flight data analysis. All emulsion films were scanned by latest emulsion scanning system, HTS [12]. For converter, $\gamma$-ray event detection processing is going on. $\gamma$-rays coming from hadron interactions in the converter and $\gamma$-rays coming from a launching plate were confirmed. For timestamper, major improvements and well-worked all stages were confirmed. Timestamp processing is going on. A timing resolution of sub-second which was enough for 1 degree imaging resolution $(>100 \mathrm{MeV})$ aimed in this experiment was evaluated with hadron interaction tracks. For attitude monitor, complementary attitude monitoring by 3 star cameras with each 90 degree azimuth was performed. A fraction of attitude determination of $98.9 \%$ and enough attitude monitoring accuracy within 0.022 degree were achieved for a Vela pulsar obsevation. $\gamma$-ray event timestamping is going on. Emulsion track timing and attitude combining (arrival direction reconstructing) is also going on. By reconstruction of arrival direction, east-west effect in horizontal coordinates was confirmed and 


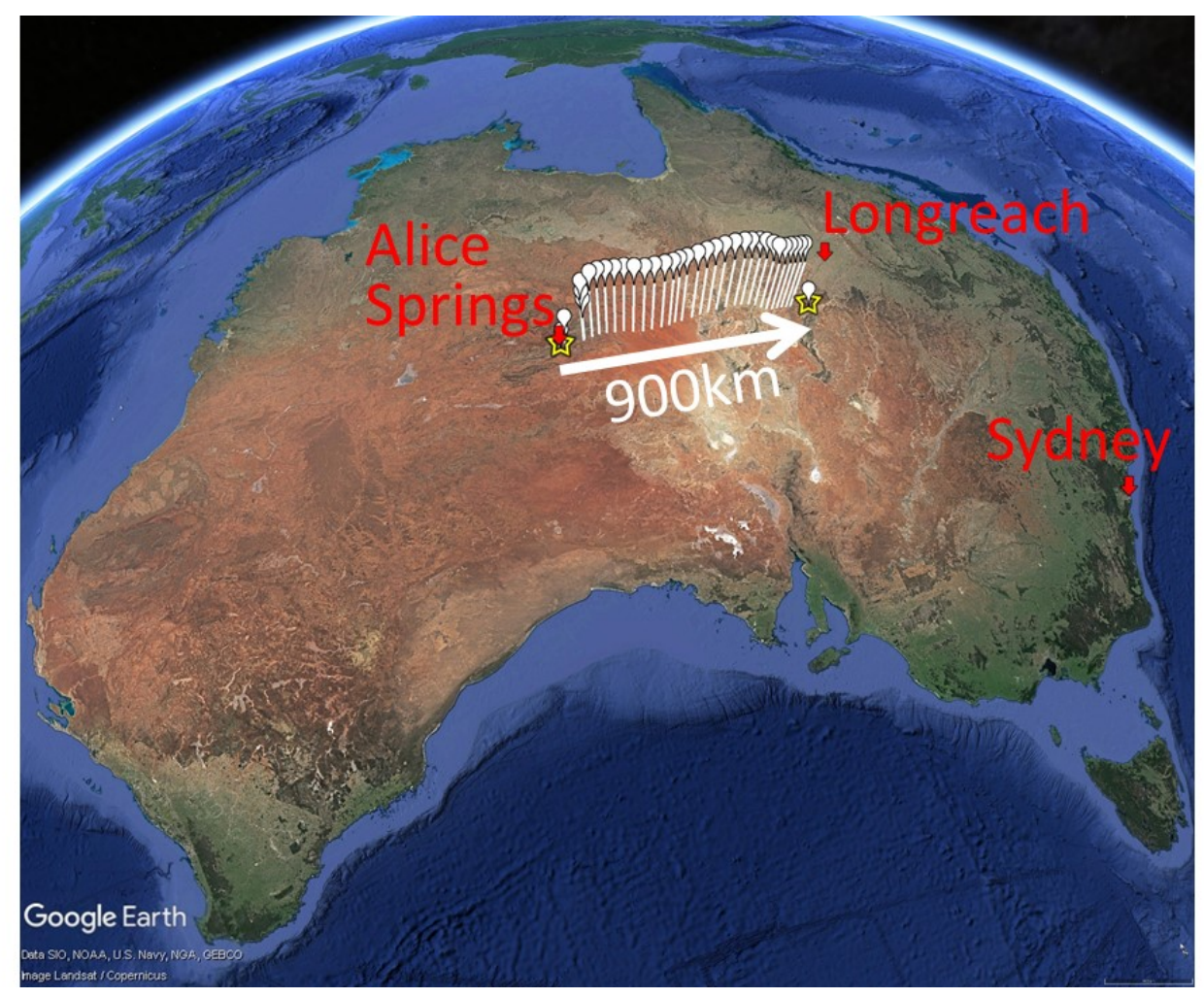

Figure 5: Flight path.

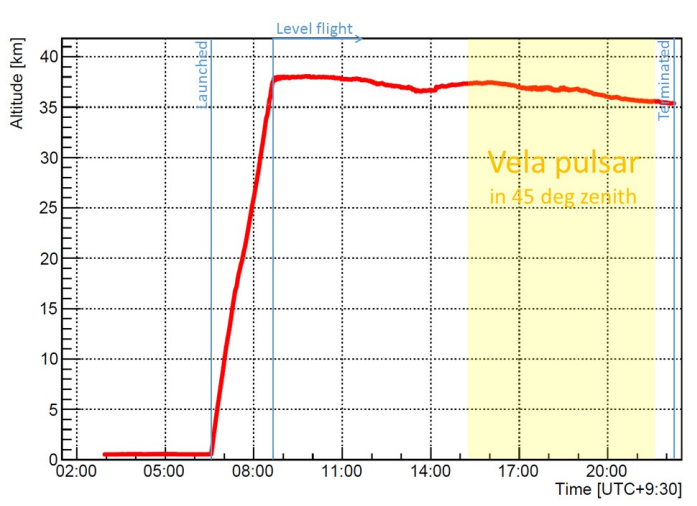

Figure 6: Altitude.

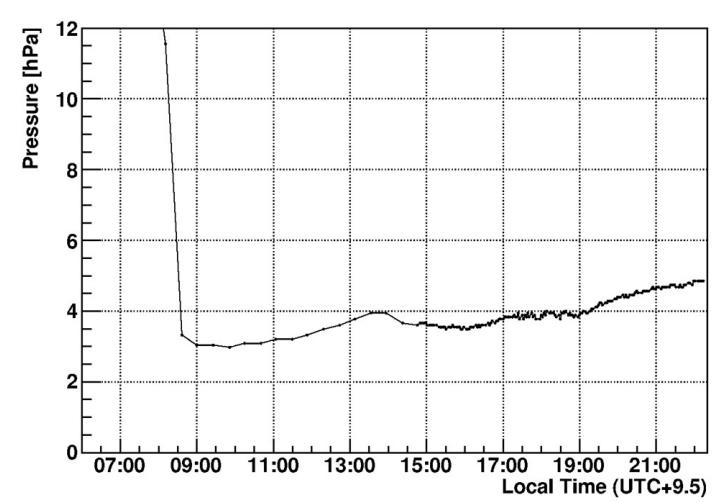

Figure 7: Residual atmospheric pressure. 


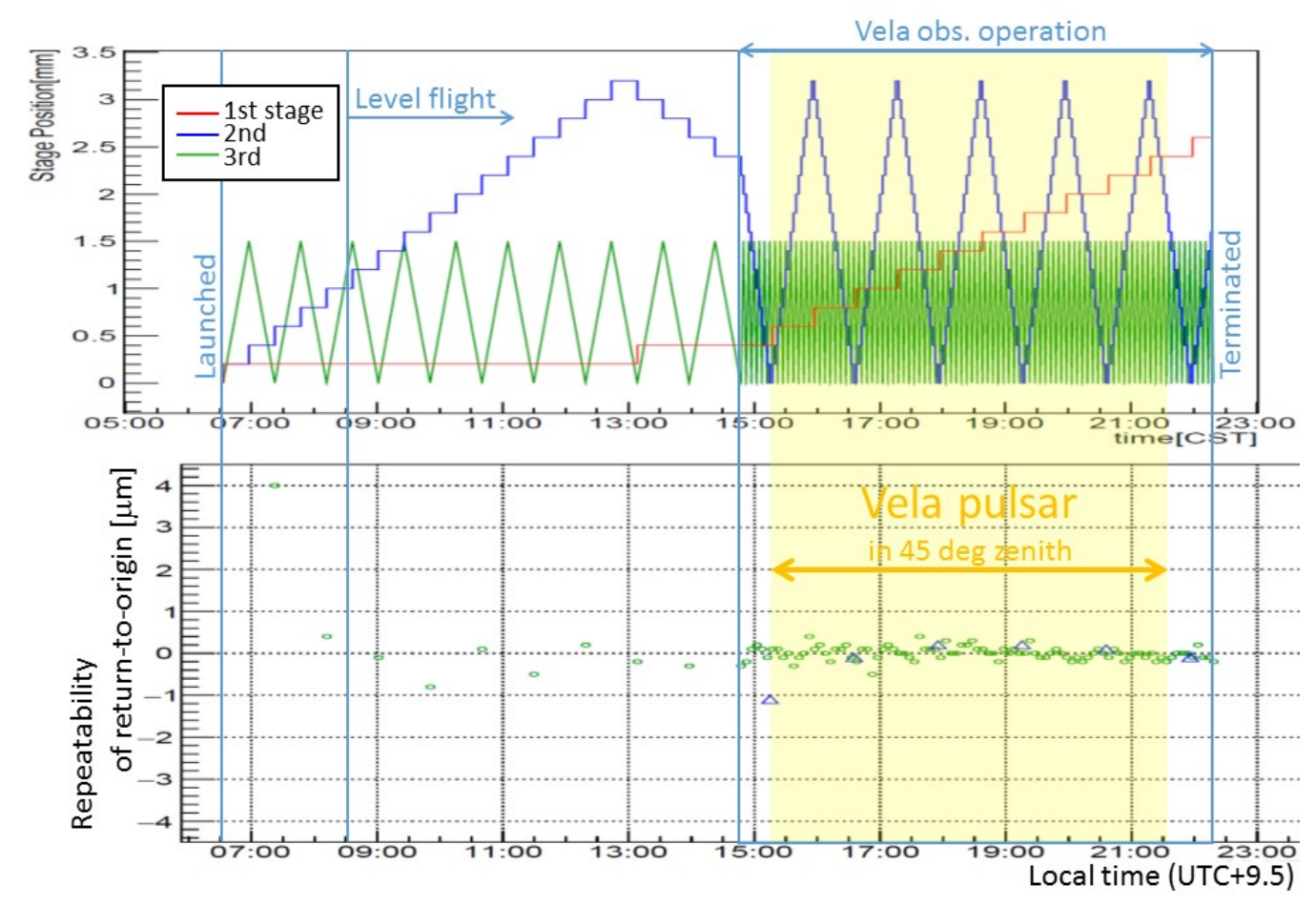

Figure 8: Operation (top) and stability (bottom) of multi-stage shifter.

arrival direction reconstruction was demonstrated for the first time in the emulsion $\gamma$-ray telescope. Reconstruction of $\gamma$-ray arrival direction in celestial coordinates is going on.

Major improvements for an effective area $\times$ time and background from the 2015 balloonborne experiment are being obtained with a total of 5 times $(\sqrt{5}$ times for detection sensitivities to $\gamma$-ray sources). Analyzing the flight data then detecting and imaging the Vela pulsar, we aim to demonstrate overall performance of the telescope.

\section{Future prospects}

In 2021 or later, with a $10 \mathrm{~m}^{2}$ and $>\sim 24$ hour flight duration, we aim to start scientific observations [14].

\section{Acknowledgments}

Emulsion film processing post-flight was supported by A. Bakich, K. Varvell, D. Beech, N. Ioannidis, and others at the University of Sydney. The final preparation and launch was done at the Alice Springs balloon-launching station managed by R. Sood and others at the University of New South Wales. The balloon-borne experiment was conducted by the Scientific Ballooning (DAIKIKYU) Research and Operation Group, ISAS, JAXA. This work was supported by JSPS KAKENHI (Grant Numbers 17H06132, 18H01228 and 18K13562). 


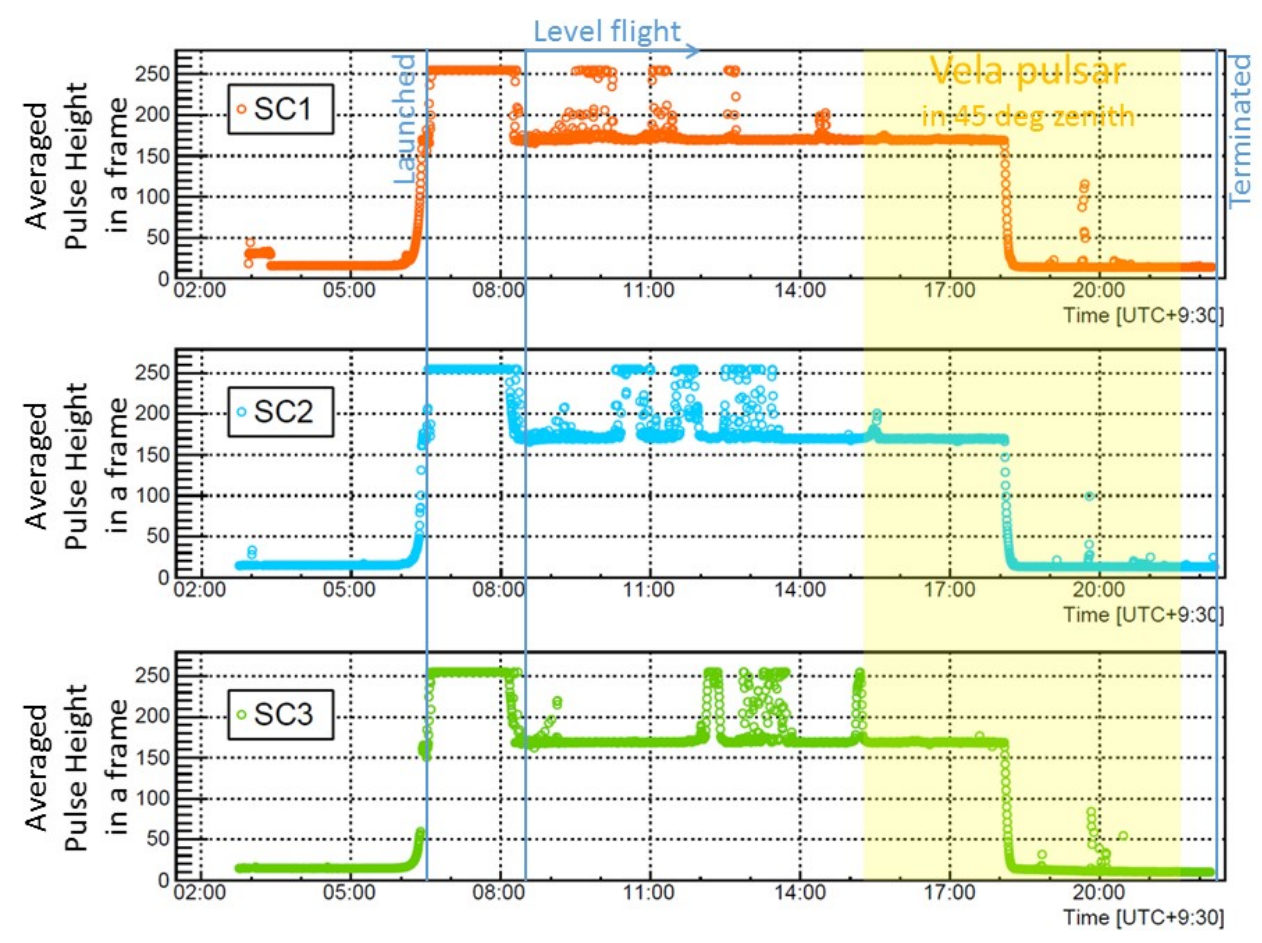

Figure 9: Operations of star cameras.

\section{References}

[1] Satoru Takahashi, Shigeki Aoki, Hiroki Rokujo, Kaname Hamada, Masahiro Komatsu, Kunihiro Morishima, Mitsuhiro Nakamura, Toshiyuki Nakano, Kimio Niwa, Osamu Sato, Teppei Yoshioka, Koichi Kodama, Time stamp technique using a nuclear emulsion multi-stage shifter for gamma-ray telescope, Nucl. Instrum. Meth. A 620 (2010) 192.

[2] S. Takahashi, A Development of Cosmic $\gamma$-Ray Telescope with Nuclear Emulsion, Ph.D. Thesis, Nagoya University, Japan, 2011 (in Japanese).

[3] Keita Ozaki, Satoru Takahashi, Shigeki Aoki, Keiki Kamada, Taichi Kaneyama, Ryo Nakagawa, Hiroki Rokujo, Demonstration of polarization sensitivity of emulsion-based pair conversion telescope for cosmic gamma-ray polarimetry, Nucl. Instr. And Meth. A 833 (2016) 165.

[4] Satoru Takahashi, Shigeki Aoki, Keiki Kamada, Saki Mizutani, Ryo Nakagawa, Keita Ozaki, and Hiroki Rokujo, GRAINE project: The first balloon-borne, emulsion gamma-ray telescope experiment, Prog. Theor. Exp. Phys. 043H01 (2015).

[5] H. Rokujo, S. Aoki, S. Takahashi, K. Kamada, S. Mizutani, R. Nakagawa, K. Ozaki, Multi-stage shifter for subsecond time resolution of emulsion gamma-ray telescopes, Nucl. Instr. And Meth. A 701 (2013) 127.

[6] S. Takahashi et al., GRAINE 2015, a balloon-borne emulsion $\gamma$-ray telescope experiment in Australia, Prog. Theor. Exp. Phys. 073F01 (2016). 


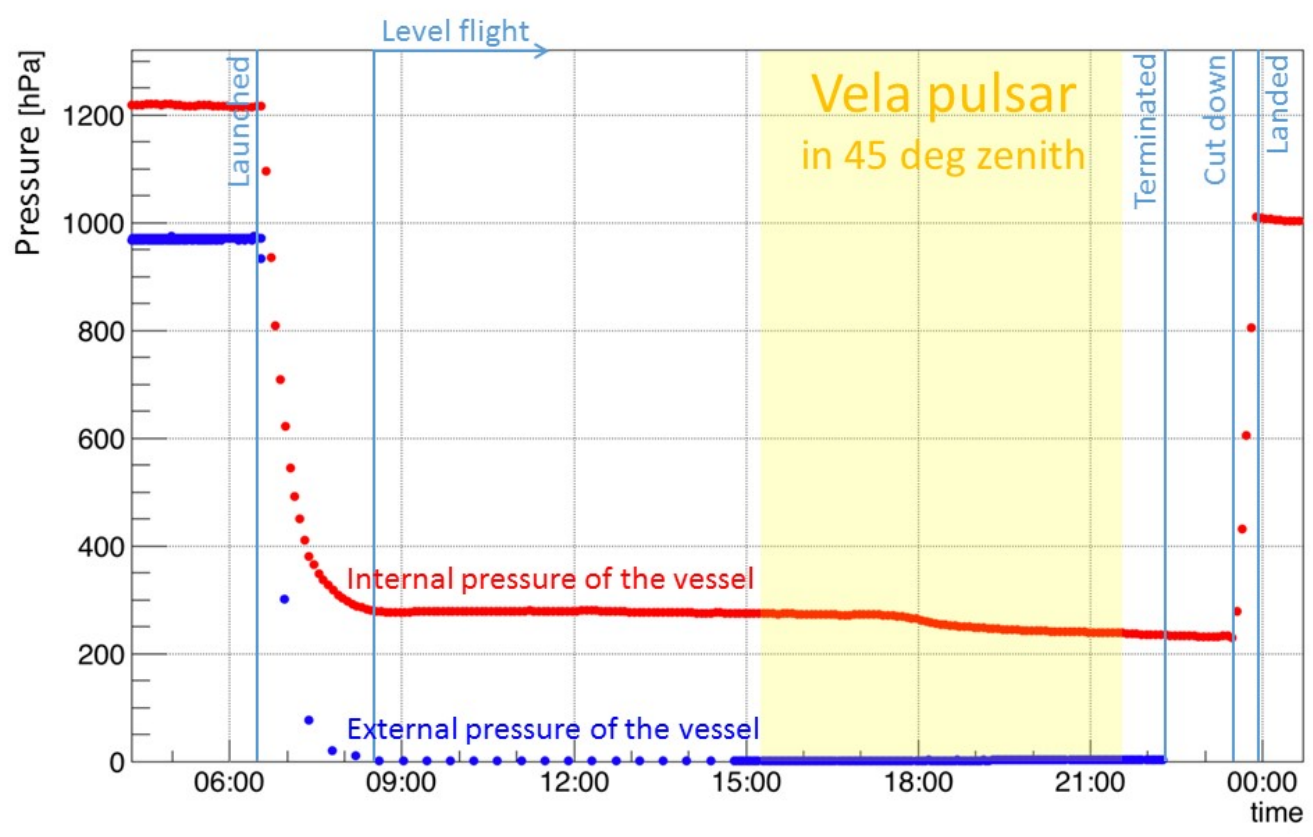

Figure 10: Internal and external pressure of the vessel.

[7] K. Ozaki, S. Aoki, K. Kamada, T. Kosaka, F. Mizutani, E. Shibayama, S. Takahashi, Y. Tateishi, S. Tawa, K. Yamada, H. Kawahara, N. Otsuka and H. Rokujo, Development of new-type nuclear emulsion for a balloon-borne emulsion gamma-ray telescope experiment, JINST 10 P12018 (2015).

[8] H. Rokujo, et al., First demonstration of gamma-ray imaging using a balloon-borne emulsion telescope, Prog. Theor. Exp. Phys. 063H01 (2018).

[9] Shigeki Aoki, Satoru Takahashi, Hiroki Rokujo, et al., GRAINE project, precise observations of high-energy cosmic $\gamma$-rays, RADIOISOTOPES (submitted).

[10] K. Yamada et al., First demonstration of an emulsion multi-stage shifter for accelerator neutrino experiments in J-PARC T60, Prog. Theor. Exp. Phys. $063 \mathrm{H} 02$ (2017).

[11] H. Rokujo et al., Development of a balloon-style pressure vessel gondola for balloon-borne emulsion gamma-ray telescopes, JINST (submitted).

[12] Masahiro Yoshimoto, Toshiyuki Nakano, Ryosuke Komatani, Hiroaki Kawahara, Hyper-track selector nuclear emulsion readout system aimed at scanning an area of one thousand square meters, Prog. Theor. Exp. Phys. 103H01 (2017).

[13] H.Rokujo et al., Gamma-ray Imaging Performance of Nuclear Emulsion Telescope in GRAINE-2018 Balloon Experiment, Proc. 36rd Int. Cosmic Ray Conference PoS(ICRC2019)596.

[14] S. Takahashi, S. Aoki, et al., GRAINE project, prospects for scientific balloon-borne experiments, Adv. Sp. Res. 62 (2018) 2945. 